\title{
The Possible Beneficial Impacts of Evodiamine on Liver Toxicity Induced by Experimental Cisplatin Administration: Effects on Oxidative Stress, Inflammation and Apoptosis
}

Fazile Nur Ekinci Akdemir ( $\sim$ fazilenur85@gmail.com)

Agri Ibrahim Cecen University: Agri Ibrahim Cecen Universitesi https://orcid.org/0000-0001-9585-3169

Serkan Yıldırım

Ataturk University: Ataturk Universitesi

Fatih Mehmet Kandemir

Ataturk University: Ataturk Universitesi

\section{Research Article}

Keywords: Apoptosis, cisplatin, evodiamine, liver toxicity, oxidative stress

Posted Date: February 4th, 2022

DOI: https://doi.org/10.21203/rs.3.rs-1234799/v1

License: (1) (i) This work is licensed under a Creative Commons Attribution 4.0 International License.

Read Full License 


\section{Abstract}

In this study, it was aimed to determine the possible beneficial effects of evodiamine on liver toxicity induced by experimental cisplatin administration in rats. For this purpose, experimental animals were divided into four groups $(n=6)$. Groups were designed as control, evodiamine (EVO), cisplatin (CIS) and evodiamine+cisplatin (EVO+CIS) groups. All experimental process was applied according to rules of ethical. Rats were sacrificed by high dose anaesthesia. The considering the biochemical results of this study, it can be said that lipid peroxidation level increased and antioxidant enzyme activities decreased in the CIS group comparing to control and only EVO groups. But in EVO+CIS group, antioxidant activities increased and lipid peroxidation decreased. Moreover, immunohistochemically caspase 8 and TNF-a expressions were severe in CIS group, whereas, in EVO+CIS group, these expressions attenuated. According to all our findings, it can be expressed that evodiamine has beneficial effects against hepatotoxicity induced by experimental cisplatin administration

\section{Introduction}

Cisplatin is a chemotherapeutic agent with an organic platinum derivative used in the treatment of a wide variety of cancers (Bentli et al. 2013, Cagin et al. 2015). However, this wide use of cisplatin with its antitumoral effect causes many undesirable effects such as hepatotoxicity, brain toxicity, ototoxicity and nephrotoxicity (Ekinci-Akdemir et al. 2017, Ekinci Akdemir et al. 2017, Kilic et al. 2018, Sakat et al. 2018). It has been determined that cisplatin leads to hepatotoxicity development at different (low or high) doses (Zicca et al. 2002, Dkhil et al. 2013, Ekinci Akdemir et al. 2017). In various studies, it has been put forward that the mechanism of hepatotoxicity induced by cisplatin can be different factors. Especially oxidative stress caused by free radicals has a significant place among these factors (Palipoch et al. 2014, Cagin et al. 2015).

Evodiamine is a natural product obtained from plants like Tetradium and shown as harmless to human health (Kobayashi et al. 2001). It has been preferred for a long time in traditional Chinese herbal medicine for the therapy of such situations of vomiting, pyrexia and pain. Evodiamine has been reported to have anti-inflamatuar, antiobesity, anti-nociceptive effects, inhibitory and antitumor activity on a variety of cancers such as lung, liver, thyroid by activation of caspase, induction of apoptosis (Chiou et al. 1992, Kobayashi et al. 2001, Takada et al. 2005, Chen et al. 2010, Lin et al. 2016, Qiu et al. 2016).

As a result of our literature review, it could not find any experimental study investigating the effect of evodiamine on the improvement of cisplatin-induced liver damage. In this respect, in this study, we aimed to investigate the possible beneficial effect of evodiamine against hepatotoxicity due to experimental cisplatin administration.

\section{Material And Methods}

\subsection{Ethical Approval}


This study was started with the approval of Atatürk University Animal Experiments Local Ethics Committee (26.10.2017/144) and all experimental procedures were made in accordance with relevant

guidelines and regulations. All experimental stages of our study were completed in Atatürk University's Animal Experimental Application and Research Center (ATADEM). Also, biochemical, histopathological and immunohistochemical analyzes were performed in Ataturk University Veterinary Faculty Biochemistry and Pathology Laboratory.

\subsection{Animals and Experimental Procedures}

In this study, 200-220 g twenty-four Wistar albino rats were used. All animals were kept in appropriate laboratory conditions (12/12 light: night cycle, 55\% moisture, 25-degree temperature) and fed with standard pellet feed and tap water. Just before starting the experiment, all animals were weighed and randomly divided into four groups: control, evodiamine (EVO), cisplatin (CIS) and evodiamine+cisplatin $(E V O+C I S)$. No medication was applied to the animals in the control group. Evodiamine (EVO) was administered intraperitoneally at a dose of $40 \mathrm{mg} / \mathrm{kg}$ in for a week only to the animals in the evodiamine group. A $10 \mathrm{mg} / \mathrm{kg}$ single dose of cisplatin was administered intraperitoneally to the animals in the cisplatin (CIS) group. In the EVO+CIS group, $40 \mathrm{mg} / \mathrm{kg}$ of evodiamine and $10 \mathrm{mg} / \mathrm{kg}$ of cisplatin were administered intraperitoneally. Furthermore, the doses of cisplatin (Ekinci-Akdemir et al. 2017) and evodiamine (Zhao et al. 2015) used in our study were selected with reference to the doses used in previous studies. On the last day of the experiment, all animals were sacrificed by high dose anaesthesia and then rapidly removed the liver tissue. Hepatic tissues were maintained under appropriate conditions until biochemical, immunohistochemical and histopathological evaluations were made.

\subsection{Biochemical Analyses}

All biochemical procedures were performed in Biochemistry Laboratory of Veterinary Faculty, Atatürk University. After the tissues were homogenized, superoxide dismutase (Sun et al. 1988), catalase (Aebi 1984), glutathione (Sedlak and Lindsay 1968), glutathione peroxidase (Lawrence and Burk 2012) enzyme activities and malondialdehyde level (Placer et al. 1966) were analyzed by using different methods in supernatants obtained from homogenates.

\subsection{Histopathologic and Immunohistochemical Evaluations}

Hepatic tissue samples received for histopathological assessment were fixed in $10 \%$ formalin solution during 48 hours. Tissue samples were embedded in the resulting paraffin blocks using standard tissue follow-up procedures. Cross sections were taken from each block with a thickness of $4 \mu \mathrm{m}$. Samples arranged for histopathological assessment were stained with hematoxylin-eosin (HE) and examined using light microscopy (Leica DM 1000, Germany). The sections were appraised by scoring according to their immunity positivity as no $(-)$, mild (+), moderate $(++)$ and severe $(+++)$.

For immunoperoxidase examination, all sections were dehydrated by passing through xylol and alcohol series and washed into distilled water for $5 \mathrm{~min}$. The sections were exposed to microwave oven 4 times 
for 5 minutes in an antigen retrieval (citrate buffer, $\mathrm{pH}$ 6.1) solution to prevent masking of the antigen in the core, then removed from the microwave oven and allowed to cool to room temperature for 30 minutes. At the end of this duration, sections were washed with distilled water, dried around the sections and drawn with special glass pencil. Endogenous peroxidase was inactivated keeping in $3 \%$ hydrogen peroxide for 10 min by washing with phosphate buffered solution (PBS, pH 7.2) for 5 min. After washing in PBS for 5-10 min, it was incubated for 5 min with Protein Block compatible with all primer and secondary antibody to prevent nonspecific ground staining. To expose the antigen in the tissues, the antigen was allowed to cool with the retrieval solution in a microwave oven for $2 \times 5$ min at 500 watts. Tissues were incubated with caspase 8 and Tnf- $\alpha$ as an apoptotic marker (Catalog no: SC5263, SC52746 Santa Cruz, USA), for $60 \mathrm{~min}$ at $37^{\circ} \mathrm{C}$.. It was followed as the immunohistochemistry kit procedure (AbcamHRP / DAB Detection IHC kit). 3-3 (Diaminobenzidine (DAB) was used as the chromogen. The staining was performed with hematoxylin. The immunohistochemical and histopathological methods used in this study were performed similarly according to the method used in previous studies (EkinciAkdemir et al. 2018, Ekinci Akdemir et al. 2019, Ekinci Akdemir et al. 2019). The sections were evaluated as no $(-)$, mild (+), moderate $(++)$ and severe $(+++)$ according to their immunity positivity.

\subsection{Statistical Analyses}

Kruskal-Wallis and Mann-Whitney $U$ tests were used for the analysis of differences between groups of obtained semiquantitatively data. Furthermore, all the biochemical data obtained in our study were analyzed by applying One-Way ANOVA and Tukey HSD tests. All statistical data were introduced as Mean $\pm S E M$ with minimum and maximum values. Statistical significance level was adopted as $p<0.05$.

\section{Results}

\subsection{Biochemical Results}

When the malondialdehyde level and antioxidant enzyme activities of the hepatic tissues are evaluated, it can be seen that it causes serious damage on the liver tissue causing lipid peroxidation and an excessive amount of free radical formation in the tissue in cisplatin induction according to control group. In addition, it was observed in the CIS group that the antioxidant system was insufficient in free radical scavenging of liver tissue due to excessive free radical formation. However, in the EVO+CIS group, the amount of malondialdehyde decreased compared to the CIS group, but the antioxidant enzyme activity increased (see Table 1).

\subsection{Histopathologic Results}

The histological appearance of liver tissues of the rats in the control group was normal (Figure 1-A). In liver tissues of the cisplatin group, severe hydropic degeneration and coagulation necrosis was detected in hepatocytes in the acinar region, while severe hyperemia in vessels and sinusoids was detected (Figure 1-B). In the evodiamine group, the liver tissues of rats were found to have normal histological structure (Figure 1-C). In the EVO+CIS group, a mild hydropic degeneration was observed in hepatic tissues, 
hepatocytes in the acinar region and necrotic hepatocytes were not observed (Figure 1D). Histopathological findings are summarized in Table 2.

\subsection{Immunohistochemical Results}

In the control and evodiamine groups, liver tissue caspase 8 and Tnf-a expression were found to be negative (Figure 2,3-A,C). In the immunohistochemical examination of liver tissues of the cisplatin group, it was revealed severe cytoplasmic caspase 8 expressions in hepatocytes (Figure 2-B). Severe Tnf-a expressions were observed in the wall of the sinusoids and in the wall of the vessels (Figure 3-B). In the EVO+CIS group, immunohistochemically, slight expression of caspase 8 was detected in hepatocytes (Figure 2-D). A slight Tnf-a expression was observed around the sinusoid and vein (Figure 3-D). Immunohistochemical findings are summarized in Table 2.

\section{Discussion}

The liver is an organ with complex functions that it has many physiological functions. While many drugs are metabolized by the liver, liver tissue is direct and/or indirectly affected in many clinical conditions or diseases (Su et al. 2018). There are many studies showing that antineoplastic drugs or chemotherapeutic agents cause direct toxicity on the liver (Ekinci Akdemir et al. 2017, Ekinci-Akdemir et al. 2018). Although there is no scientific data clearly demonstrating the cause of cisplatin-induced liver toxicity, most of the researchers focus on the theory of free radical formation and oxidative stress in cisplatin-induced liver toxicity (Cagin et al. 2015, Ekinci Akdemir et al. 2017, Ekinci-Akdemir et al. 2018). The liver is a vital detoxification organ in the organism and is primarily affected by the toxic effects of drugs (Maes et al. 2016). Liver toxicity, high doses of cisplatin or application of low recurrence doses, probably due to cumulative accumulation in liver tissue is reported to be shaped (Cvitkovic 1998, Fenoglio et al. 2005, Iraz et al. 2006, Pratibha et al. 2006). Cisplatin causes disruption of energy metabolism, lipid peroxidation and oxidative damage by altering ATP and glutathione concentration through mitochondria in the liver (Martins et al. 2008). Production and cleaning of reactive oxygen species (ROS) in cells under normal physiological conditions are regulated by endogenous defence systems such as catalase (CAT), superoxide dismutase (SOD) and glutathione (GSH). In the case of oxidative stress, the ROS level increases and the cellular structures such as protein, lipid and DNA are damaged (Ekinci Akdemir and Tanyeli 2019). ROS has an important function in the pathogenesis of cisplatin-induced damage (Dasari and Tchounwou 2014, Ekinci Akdemir et al. 2017, Katanic et al. 2017). Because of the cisplatin can be bound with cellular proteins, it leaves the cell vulnerable via also inhibit enzymes such as CAT and SOD, which can protect the cells against oxidative damage by affecting the structure of antioxidant enzymes (Almaghrabi 2015, Katanic et al. 2017). Made in this regard studies have shown that oxidative stress parameters (CAT, SOD, GSH and TBARS) in the liver and various tissues vary significantly in cisplatintreated experimental animals and as a result, cisplatin treatment effects as unfavourable the organs (Ekinci-Akdemir et al. 2017, Ekinci Akdemir et al. 2017, Katanic et al. 2017). Also, it has been revealed that cisplatin treatment significantly decreased antioxidant enzymes and GSH levels in the liver and different tissues, however, TBARS/MDA level and lipid peroxidation increased significantly (Ekinci-Akdemir et al. 
2017, Ekinci Akdemir et al. 2017, Katanic et al. 2017). When the results of oxidative stress parameters (MDA, GSH, GPx, SOD and CAT) of this study were evaluated, as the similar to the results of previous studies, it was seen that MDA level was increased and antioxidant enzyme activities decreased significantly due to cisplatin. However, as can be seen in our findings, treatment with eugenol decreased level of the oxidative damage caused by cisplatin.

Apoptosis can be initiated by a variety of factors, including toxic substances, chemotherapeutic treatments, ionizing radiation, and oxidants. ROS formation and oxidative damage play an important role in the initiation of apoptosis. Apoptosis can also be induced by oxidative stress due to damage to both the mitochondria, the plasma membrane and the core material. ROS, oxidative damage and apoptosis have been reported to have strong relationships with each other (Kiess and Gallaher 1998, Qu et al. 2001). The caspase family is primarily or indirectly responsible for all stages of apoptotic pathways in living cells. Hence, activities of the caspase enzymes and procaspases are the most prominent actors of apoptotic cell death (Thornberry 1997, Julien and Wells 2017). Cell death proteases, known as caspases, are integral components of different types of apoptotic programs (Salvesen and Dixit 1997). Caspases have widely existed in inactive proenzyme form in cells. Once activated, they activate other procaspases, which allow starting a protease cascade. This proteolytic cascade, in which a caspase can activate the other caspase, increases the apoptotic signalling pathway and thus leads to rapid cell death. Caspase 8 plays an important role in the initiation of apoptosis (Cohen 1997, Rai et al. 2005). In previous studies, it has been demonstrated that the level of TNF-a from proinflammatory cytokines is increased in the organ toxicity induced by cisplatin (Abdelrahman et al. 2019). Caspase 8 and TNF-a expressions were significantly exacerbated by cisplatin induction. In contrast, the expressions of caspase and cytokine were decreased by evodiamine treatment.

In addition to the biochemical results, in the previous studies done on cisplatin or other chemotherapeutic drugs-induced liver tissue toxicity in experimental animals, it is revealed the pathological tissue damage characterized by degenerated hepatocytes and significant changes in liver morphology including obstructions, vacuolization in sinusoidal, dilatation of the vessels, severe activation of Kupffer cells and etc (Iseri et al. 2007, Omar et al. 2016, Ekinci-Akdemir et al. 2018). In view of the histopathological findings presented in our study as accordance with the studies in the literature, it was observed that a significant degeneration in hepatocytes such as necrosis, dilatation and hyperemia. However, evodiamine treatment was found to decrease pathological tissue damage. When the findings of the oxidative and antioxidant parameters, histopathological and immunohistochemical parameters were examined, it was evaluated that the evodiamine has a beneficial effect on liver tissue damage induced by cisplatin.

\section{Declarations}

\section{Conflict of interest}

The authors declared that there is no conflict of interest.

\section{Acknowledgements}


This study was funded by Ağrı İbrahim Çeçen University Scientific Research Projects Unit (Project Number: SY0.18.002).

\section{Disclosure statement}

No potential conflict of interest was reported by the authors.

\section{Funding}

This study was funded by A_grı_Ibrahim C,ec,en University Scientific Research Projects Unit [Project Number: SY0.18.002].

\section{Ethical approval}

All applicable international, national, and/or institutional guidelines for the care and use of animals were followed. This study was approved of Ataturk University Experimental Animals Local Ethics Committee (26.10.2017/144).

\section{Consent to Publish}

Not applicable

\section{Consent to Participate}

Not applicable

\section{References}

1. Abdelrahman AM et al (2019) Effect of levosimendan, a calcium sensitizer, on cisplatin-induced nephrotoxicity in rats. Toxicology Reports 6:232-238

2. Aebi H (1984) Catalase Invitro Methods Enzymology 105:121-126

3. Almaghrabi OA (2015) Molecular and biochemical investigations on the effect of quercetin on oxidative stress induced by cisplatin in rat kidney. Saudi Journal of Biological Science 22(2):227231

4. Bentli R et al (2013) Molsidomine prevents cisplatin-induced hepatotoxicity. Archieves of Medical Research 44(7):521-528

5. Cagin YF et al (2015) Protective Effects of Apocynin on Cisplatin-induced Hepatotoxicity in Rats. Archieves of Medical Research 46(7):517-526

6. Chen MC et al (2010) Anti-proliferative effects of evodiamine on human thyroid cancer cell line ARO. J Cell Biochem 110(6):1495-1503

7. Chiou WF et al (1992) The vasorelaxant effect of evodiamine in rat isolated mesenteric arteries: mode of action. Eur J Pharmacol 215(2-3):277-283 
8. Cohen GM (1997) Caspases: the executioners of apoptosis. Biochem J 326:1-16

9. Cvitkovic $E$ (1998) Cumulative toxicities from cisplatin therapy and current cytoprotective measures. Cancer Treat Rev 24(4):265-281

10. Dasari S, Tchounwou PB (2014) Cisplatin in cancer therapy: Molecular mechanisms of action. Eur J Pharmacol 740:364-378

11. Dkhil MMA et al (2013) The potential role of Azadirachta indica treatment on cisplatin-induced hepatotoxicity and oxidative stress in female rats. Oxidative Medicine and Cellular Longevity, 2013, 741817

12. Ekinci-Akdemir FN et al (2017) Effect of P-Coumaric Acid against Oxidative Stress Induced by Cisplatin in Brain Tissue of Rats. The Journal of Animal and Plant Science 27(5):1560-1564

13. Ekinci-Akdemir FN et al (2018) The effects of casticin and myricetin on liver damage induced by methotrexate in rats. Iranian Journal of Basic Medical Science 21(12):1281-1288

14. Ekinci Akdemir FN et al (2017) The Protective Effects of p-Coumaric Acid on Acute Liver and Kidney Damages Induced by Cisplatin.Biomediciens, 5(2)

15. Ekinci Akdemir FN, Tanyeli A (2019) The Antioxidant Effect of Fraxin against Acute Organ Damage in Polymicrobial Sepsis Model induced by Cecal Ligation and Puncture. Turkish Journal of Science $4(1): 22-29$

16. Ekinci Akdemir FN et al (2019) The antiapoptotic and antioxidant effects of eugenol against cisplatin-induced testicular damage in the experimental model. Andrologia, Inpress, e13353

17. Ekinci Akdemir FN et al (2019) Protective effects of gallic acid on doxorubicin-induced cardiotoxicity; an experimantal study.Archieves of Physiology and Biochemistry,1-8

18. Fenoglio $C$ et al (2005) Morphological and histochemical evidence of the protective effect of procainamide hydrochloride on tissue damage induced by repeated administration of low doses of cisplatin. Anticancer Res 25(6b):4123-4128

19. Iraz M et al (2006) Protective effect of caffeic acid phenethyl ester (CAPE) administration on cisplatin-induced oxidative damage to liver in rat. Cell Biochemistry Function 24(4):357-361

20. Iseri $S$ et al (2007) Simvastatin attenuates cisplatin-induced kidney and liver damage in rats. Toxicology 230(2-3):256-264

21. Julien O, Wells JA (2017) Caspases and their substrates. Cell Death Differentiation 24(8):1380-1389

22. Katanic $J$ et al (2017) Filipendula ulmaria extracts attenuate cisplatin-induced liver and kidney oxidative stress in rats: In vivo investigation and LC-MS analysis. Food Chem Toxicol 99:86-102

23. Kiess W, Gallaher B (1998) Hormonal control of programmed cell death apoptosis. Eur J Endocrinol 138(5):482-491

24. Kilic K (2018) Protective effect of gallic acid against cisplatin-induced ototoxicity in rats. Braz J Otorhinolaryngol 85(3):267-274

25. Kobayashi $Y$ et al (2001) Capsaicin-like anti-obese activities of evodiamine from fruits of Evodia rutaecarpa, a vanilloid receptor agonist. Planta Med 67(7):628-633 
26. Lawrence RA, Burk RF (2012) Glutathione peroxidase activity in selenium-deficient rat liver (Reprinted From Biochemical And Biophysical Research Communications, vol 71, pg 952-958, 1976). Biochemical and Biophysical Research Communucations, 425(3), 503-509

27. Lin L et al (2016) Effect of evodiamine on the proliferation and apoptosis of A549 human lung cancer cells. Mol Med Rep 14(3):2832-2838

28. Maes $\mathrm{M}$ et al (2016) Experimental models of hepatotoxicity related to acute liver failure. Toxicology Applied Pharmacology 290:86-97

29. Martins NM et al (2008) Cisplatin induces mitochondrial oxidative stress with resultant energetic metabolism impairment, membrane rigidification and apoptosis in rat liver. $\mathrm{J}$ Appl Toxicol 28(3):337-344

30. Omar HA et al (2016) Hesperidin alleviates cisplatin-induced hepatotoxicity in rats without inhibiting its antitumor activity. Pharmacological Reports 68(2):349-356

31. Palipoch $S$ et al (2014) Hepatoprotective effect of curcumin and alpha-tocopherol against cisplatininduced oxidative stress. BMC Complement Altern Med 14:111

32. Placer ZA et al (1966) Estimation of products of lipid peroxidation (as malondialdehyde) in biochemical systems. Anal Biochem 16:359-364

33. Pratibha R et al (2006) Enzymatic studies of cisplatin induced oxidative stress in hepatic tissue of rats. Eur J Pharmacol 532(3):290-293

34. Qiu C et al (2016) A promising antitumor activity of evodiamine incorporated in hydroxypropyl-betacyclodextrin: pro-apoptotic activity in human hepatoma HepG2 cells. Chem Cent J 10:46

35. Qu W (2001) Mechanisms of arsenic-induced cross-tolerance to nickel cytotoxicity, genotoxicity, and apoptosis in rat liver epithelial cells. Toxicol Sci 63(2):189-195

36. Rai NK et al (2005) Apoptosis: a basic physiologic process in wound healing. Int J Low Extrem Wounds 4(3):138-144

37. Sakat MS et al (2018) The effectiveness of eugenol against cisplatin-induced ototoxicity. Brazilian Journal of Otorhinolaryngology, In press

38. Salvesen GS, Dixit VM (1997) Caspases: Intracellular signaling by proteolysis. Cell 91(4):443-446

39. Sedlak J, Lindsay RH (1968) Estimation of total, protein-bound, and nonprotein sulfhydryl groups in tissue with Ellman's reagent. Anal Biochem 24:192-205

40. Su W et al (2018) Role of HSD17B13 in the liver physiology and pathophysiology. Molecular and Cellular Endocrinology, In press

41. Sun Y et al (1988) A Simple Method for Clinical Assay of Superoxide-Dismutase. Clin Chem 34(3):497-500

42. Takada $Y$ et al (2005) Evodiamine abolishes constitutive and inducible NF-kappaB activation by inhibiting IkappaBalpha kinase activation, thereby suppressing NF-kappaB-regulated antiapoptotic and metastatic gene expression, up-regulating apoptosis, and inhibiting invasion. J Biol Chem 280(17):17203-17212 
43. Thornberry NA (1997) The caspase family of cysteine proteases. Br Med Bull 53(3):478-490

44. Zhao $Z$ et al (2015) Effect and mechanism of evodiamine against ethanol-induced gastric ulcer in mice by suppressing Rho/NF-small ka, CyrillicB pathway. Int Immunopharmacol 28(1):588-595

45. Zicca A et al (2002) Reduction of cisplatin hepatotoxicity by procainamide hydrochloride in rats. Eur J Pharmacol 442(3):265-272

\section{Tables}

Table 1: Mean \pm SEM and Minimum-Maximum values of the Malondialdehyde (MDA), Glutathione (GSH) levels, Glutathione Peroxidase (GPx), Superoxide Dismutase (SOD) and Catalase (CAT) activities of the control, cisplatin, evodiamine and EVO+CIS groups in liver tissues.

\begin{tabular}{|c|c|c|c|c|}
\hline & $\begin{array}{l}\text { Control } \\
\text { Group }\end{array}$ & $\begin{array}{l}\text { Evodiamine } \\
\text { (EVO) group }\end{array}$ & $\begin{array}{l}\text { Cisplatin } \\
\text { (CIS) } \\
\text { group }\end{array}$ & $\begin{array}{l}\text { EVO+CIS } \\
\text { group }\end{array}$ \\
\hline Malondialdehyde (MDA) & $22,65 \pm 0,54^{\star}$ & $22,86 \pm 0,90^{\#}$ & $30,42 \pm 0,89^{*}, \#, 9$ & $27,49 \pm 0,63^{*, \#, 9}$ \\
\hline (nmol g-1 tissue) & $(22,65 \pm 0,54)$ & $(20,22-26,11)$ & $(30,33-36,51)$ & $(25,34-29,77)$ \\
\hline Glutathione (GSH) & $3,21 \pm 0,06^{*}$ & $3,43 \pm 0,05^{\#}$ & $1,74 \pm 0,04^{\star}, \#, 9$ & $2,65 \pm 0,05^{\star}, \#, 9$ \\
\hline (nmol g-1 tissue) & $(3,01-3,45)$ & $(3,28-3,64)$ & $(1,59-1,88)$ & $(2,42-2,88)$ \\
\hline \multirow{2}{*}{$\begin{array}{l}\text { Glutathione Peroxidase (GPx) } \\
\left(\mathrm{U} \mathrm{g}^{-1} \text { protein) }\right.\end{array}$} & $14,54 \pm 0,58^{*}$ & $14,83 \pm 0,45^{\#}$ & $8,57 \pm 0,20^{*}, \#, 9$ & $11,80 \pm 0,33^{*, \#, 9}$ \\
\hline & $\begin{array}{l}(12,84- \\
16,43)\end{array}$ & $(13,03-16,33)$ & $(7,98-9,13)$ & $(10,65-13,02)$ \\
\hline \multirow{2}{*}{$\begin{array}{l}\text { Superoxide Dismutase (SOD) } \\
\left(\mathrm{U} \mathrm{g}^{-1} \text { protein }\right)\end{array}$} & $22,94 \pm 0,61^{*}$ & $23,29 \pm 1,00^{\#}$ & $15,71 \pm 0,41^{*, \#, 9}$ & $19,28 \pm 0,37^{\star}, \#, 9$ \\
\hline & $\begin{array}{l}(20,88- \\
25,04)\end{array}$ & $(20,43-26,01)$ & $(14,59-17,02)$ & $(17,84-20,11)$ \\
\hline \multirow{2}{*}{$\begin{array}{l}\text { Catalase (CAT) (Catal g-1 } \\
\text { protein) }\end{array}$} & $9,75 \pm 0,32^{*}$ & $10,11 \pm 0,58^{\#}$ & $5,31 \pm 0,23^{*}, \#, 9$ & $7,66 \pm 0,22^{*}, \#, 9$ \\
\hline & $(8,79-11,01)$ & $(8,43-12,01)$ & $(4,56-6,18)$ & $(6,95-8,53)$ \\
\hline
\end{tabular}

$*, \#$, Statistical significant is seen as $p<0.05$. There is a statistical relationship between the groups where the same symbols.

Table 2: Histopathological and immunohistochemical scoring of the control, cisplatin (CIS), evodiamine (EVO) and evodiamine + cisplatin (EVO+CIS) groups in liver tissues. 


\begin{tabular}{|c|c|c|c|}
\hline $\begin{array}{l}\text { Control } \\
\text { aroup }\end{array}$ & $\begin{array}{l}\text { Cisplatin } \\
\text { (CIS) group }\end{array}$ & $\begin{array}{l}\text { Evodiamine } \\
\text { (EVO) group }\end{array}$ & $\begin{array}{l}\text { EVO+CIS } \\
\text { group }\end{array}$ \\
\hline
\end{tabular}

$\begin{aligned} & \text { Degeneration in } \\ & \text { hepatocytes }\end{aligned}$
he

Necrosis in hepatocytes $\quad$ - $\quad++\quad-$

Hyperaemia in the vessels

$+++\quad++$

The expression of Caspase 8

The expressigures

\section{Figure 1}

Liver tissue, control group, normal histological appearance (A), cisplatin group, necrosis in hepatocytes (arrows), degeneration (arrowheads) (B), evodiamine group, normal histological appearance (C), EVO+CIS group, degeneration in hepatocytes (arrowheads) (D), H\&E, Bar: $20 \mu \mathrm{m}$.

\section{Figure 2}

Expression of liver tissue, control group, negative Caspase $8(A)$, cisplatin group, severe expression of caspase 8 in hepatocytes (arrowheads) (B), evodiamine group, negative expression of Caspase 8 (C), EVO+CIS group, hepatocytes slight expression of caspase 8 expression (arrowheads) (D), IHC-P, Bar: 20 $\mu \mathrm{m}$. 


\section{Figure 3}

Liver tissue, control group, negative Tnf-a expression (A), cisplatin group, severe Tnf-a expression level in the sinusoidal intervals and perivascular field (arrowheads) (B), evodiamine group, negative Tnf-a expression (C), EVO+CIS group, mild level Tnf-a expressions in the sinusoidal field (arrows) (D), IHC-P, Bar: $20 \mu \mathrm{m}$.

\section{Supplementary Files}

This is a list of supplementary files associated with this preprint. Click to download.

- graphicalabstract.jpg 